\title{
Liposomes modified by mono- and bis-phthalocyanines: A comprehensive EPR study
}

\author{
Dariusz Man $^{1, a}$, Rudolf Słota ${ }^{2}$, Anna Kawecka ${ }^{1}$, Grzegorz Engel ${ }^{1}$, and Gabriela Dyrda ${ }^{2}$ \\ 1 Institute of Physics, Opole University, Oleska 48, 45-052 Opole, Poland \\ 2 Faculty of Chemistry, Opole University, Oleska 48, 45-052 Opole, Poland
}

Received 6 November 2016 and Received in final form 1 May 2017

Published online: 19 June 2017

(C) The Author(s) 2017. This article is published with open access at Springerlink.com

\begin{abstract}
The impact of selected metallophthalocyanines, featuring diverse molecular structure, upon the fluidity of liposome membranes was studied using the spin label EPR technique. The "mono"-type MPc's $\left(\mathrm{M}=\mathrm{Zn}, \mathrm{Sn} ; \mathrm{Pc}=\mathrm{C}_{32} \mathrm{H}_{16} \mathrm{~N}_{8}\right.$ is the phthalocyanine ligand) and sandwich LnPc $\mathrm{c}_{2}$ complexes ( $\mathrm{Ln}=\mathrm{Nd}, \mathrm{Sm}$, $\mathrm{Gd}$ ) were explored. Liposomes were obtained in a sonication process, from egg yolk lecithin (EYL) in water. TEMPO and 16-DOXYL spin labels were used to monitor the peripheral and central part of the lipid double layer, respectively, which allowed to localize the phthalocyanine additive within the bilayer, as well as to perform independent measurements of changes in fluidity upon addition thereof. All the complexes tested were found to increase the fluidity in the middle of the lipid bilayer. However, at the water-lipid interface the $\mathrm{LnPc}_{2}$ compounds showed a relative small effect upon the phospholipids' arrangement, whereas in the case of $\mathrm{ZnPc}$ and $\mathrm{SnPc}$ it was found much more pronounced. EPR results were supplemented by measurements of static electrical charge, the investigated phthalocyanines may potentially feed into the membrane thus affecting its stability.
\end{abstract}

\section{Introduction}

Phthalocyanines, due to their physicochemical versatility have been ranked among the top important molecular materials synthesized in the XX century. There is a rich choice of published scientific work dedicated to these extraordinary molecules $[1-3]$. The chemical structure of phthalocyanine $\left(\mathrm{H}_{2} \mathrm{Pc}\right)$ and space models of typical complexes with metal ions have been shown in fig. 1(a), (b). Diverse molecular systems featuring the phthalocyanine macrocycle may be designed by introducing peripheral functional groups, axial ligands and/or coupling two or three of such units into novel independent compounds, fig. 1(c), (d). Indeed, phthalocyanines determined a new dimension in materials chemistry and opened the way to the syntheses of sophisticated molecular set-ups based on different heteromacrocyclic systems incorporating metal ions.

The phthalocyanine macrocycle includes a very stable 16-membered aromatic chromophore (core) resulting from the coupled $\pi$ bonding system $(-\mathrm{C}=\mathrm{N}-)$. Such arrangement allows a very strong absorption of photons from the UV-Vis-NIR range and consequently determines the photochemical activity of phthalocyanines. One may tune the energetics of photon-trapping by replacing the metal

\footnotetext{
a e-mail: dariusz.man@uni.opole.pl
}

ion and/or changing the architecture of the macrocyclic periphery, e.g. as shown in fig. 1(d). The energy of the absorbed photons may be transferred to other molecules present in the ambient, which may lead to the formation of new compounds, such as reactive oxygen species (ROS). In particular, the possibility of generating singlet molecular oxygen ${ }^{1} \mathrm{O}_{2}\left(\Delta_{1 \mathrm{~g}}\right)$ by photoexcited metallophthalocyanines has been considered very attractive for diverse photochemical applications [4]. One of the most important issues is related to novel medical procedures dedicated to the treatment of neoplastic diseases, involving the use of light from the UV and Vis range, which need effective dye sensitizers. Hence, phthalocyanines have been indicated as one of the most promising candidates for both photodynamic diagnostics (PDD) and therapy (PDT) [5]. Along with the recent development of intelligent drug delivery systems, introducing photoactive phthalocyanines exactly into the targeted tissue has become feasible. The currently tested methods involve diverse smart transporting systems based upon oil emulsions, lipoproteins, micelles and liposomes [6-11]. Promising results have been obtained so far regarding the transfer of phthalocyanine complexes directly into the cancered cells using liposome vesicles doped with a zinc derivative, and the concentration of $\mathrm{ZnPc}$ within the tumor was found ten-fold greater than it was determined for the adjacent healthy cells. Similar results have been reported for a number of other 


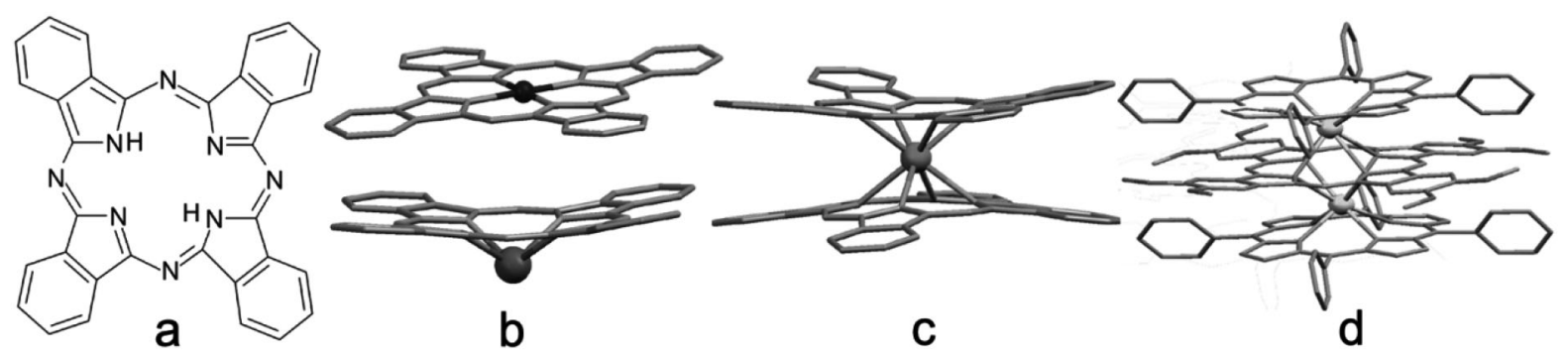

Fig. 1. Chemical and molecular structure of phthalocyanine, $\mathrm{H}_{2} \mathrm{Pc}$ (a) and its metal complexes; (b) ZnPc (top) and SnPc (bottom), (c) sandwich system of $\mathrm{NdPc}_{2}$, (d) heteroleptic compound Pp-Ce-Pc-Ce-Pp, Pp is for tetraphenylporphyrin moiety. Structures of (b)-(d) have been derived and modified based on the Cambridge Structural Database *.cif files: PTHCZN, SNPHCY01, CIZGIB02 and JUDNUR, respectively.<smiles>CC1(C)CCCC(C)(C)N1[O]</smiles>

Fig. 2. Chemical structure of the spin labels TEMPO (left) and 16-DOXYL (right). The dot mark shows the active spin position.

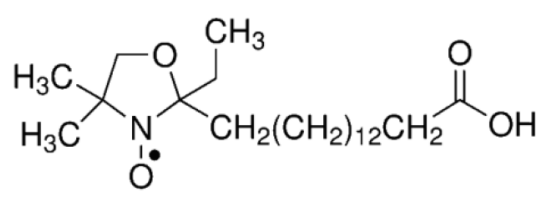

metallophthalocyanines incorporated into liposome carriers [7-9].

The stability of a phthalocyanine-liposome unit is crucial to a successful drug transport throughout a real biological system. As reported elsewhere, organometallic compounds may more or less affect the membrane's physical parameters [12-14]. Likewise, our previous investigations were focused mainly on structural deviations induced within the lipid arrangement by different amounts of such additives [15-19] including the effect of the interaction time [20,21]. On the other hand, the present work contains some novel aspects regarding the behavior of intercalated phospholipid systems, in particular the impact of the dopant's molecular structure and its static electrical charge. For this reason, the effect of selected phthalocyanine compounds on the fluidity of a model liposome membrane, obtained from EYL (egg yolk lecithin) was studied. Sandwich-type complexes $\left(\mathrm{LnPc}_{2}\right)$ of $\mathrm{Nd}, \mathrm{Sm}$ and $\mathrm{Gd}$, as well as $\mathrm{SnPc}$ and $\mathrm{ZnPc}$ representing a convex and flat molecular structure, respectively (fig. 1), were chosen to investigate the distribution of the dopant within the lipid bilayer. The electron spin resonance (EPR) method using two different spin labels, penetrating the outer phospholipid-water interface (TEMPO) and the central part of the membrane (16-DOXYL), was applied. This allowed monitoring the dynamic changes within the liposome system. Particular attention was addressed to the effect of low dopant concentration on the fluidity profile inside the phospholipid bilayer. The results obtained in this work are supposed to support the existing science concerning the molecular transport within cell membranes and consequently should help designing effective phthalocyanine-based composites for PDT applications.

\section{Materials and methods}

All solvents used (acetone, hexane, methanol, chloroform and dimethylformamide) were commercial products of analytical grade and were applied without further purification. The phthalocyanine complexes $\mathrm{ZnPc}, \mathrm{SnPc}, \mathrm{NdPc}_{2}, \mathrm{SmPc}_{2}$ and $\mathrm{GdPc}_{2}$ were homesynthesized according to the method described elsewhere [22]. Spin labels used in EPR measurements (fig. 2) TEMPO (2,2,6,6-tetramethylpiperidine-1-oxyl) and 16DOXYL (2- ethyl-2-(15-methoxy-15-oxopentadecyl)-4,4dimethyl-3-oxazolidinyloxy) were purchased from SigmaAldrich, Poland.

\subsection{Liposome formation}

Liposome vesicles were produced in a sonication process of egg yolk lecithin (EYL) in distilled water. Lecithin was separated from fresh egg yolks using acetone and hexane for extraction, and the crude product was further purified by column chromatography $\left(\mathrm{Al}_{2} \mathrm{O}_{3}\right.$ basic, chloroform: methanol as eluent) following a standard procedure [23]. The sonication of each lecithin sample $(60 \mu \mathrm{M}$ EYL and $2 \mathrm{ml} \mathrm{H}_{2} \mathrm{O}$ ) was performed with a TECHPAN UD-20 ultrasonic disintegrator in 5 cycles involving alternately $30 \mathrm{~s}$ of sonication and $60 \mathrm{~s}$ of cooling. Into such prepared liposome dispersion the appropriate spin label was added in concentration of $0.1 \%$ (molar) relative to $\mathrm{EYL}$ (i.e., molar ratio of the spin label to the lipid particles was equal 0.001) and left for 15 minutes to allow equilibration. The specified amount of the phthalocyanine solution in dimethylformamide was poured into the testing tube 
a

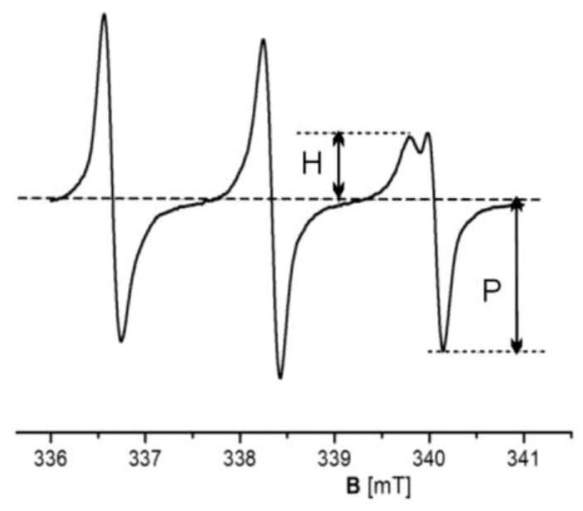

b

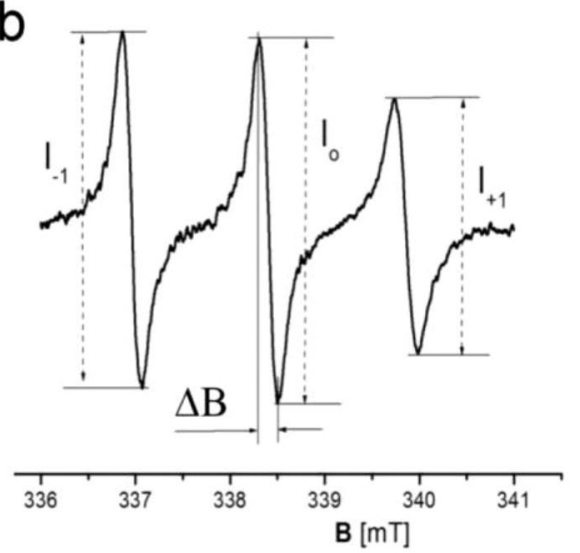

Fig. 3. EPR spectra recorded for EYL liposome dispersions in water, featuring the spectral parameters used to determine $F$ and $\tau$; (a) TEMPO and (b) 16-DOXYL spin labels.

and after solvent evaporation $0.5 \mathrm{ml}$ of the liposome dispersion was added and the content of the tube was vortexed for 5 minutes, prior to EPR measurement. The concentration of the phthalocyanine component in a doped liposome sample was gradually changed in the range of $0.1-1.2 \%$ (molar) relative to EYL.

\subsection{EPR measurements}

EPR spectra were measured at $22{ }^{\circ} \mathrm{C}$ for the EYL liposome membranes being in the liquid-crystalline state. MX-201R EPR spectrometer (UT Wrocław, Poland) was used with the following settings: $B_{0}=0.338 \mathrm{~T}$ (magnetic field center), $\Delta B=10 \mathrm{mT}$ (sweep window), $\Delta T=128 \mathrm{~s}$ (sweep time), microwave power $60 \mathrm{~mW}$, frequency $f=9.67 \mathrm{GHz}$. Two independent runs were performed in order to minimize the measurement uncertainty, and three scans were recorded for each dopant concentration. The effect of the phthalocyanine additive was evaluated based on the values of the below-defined spectroscopic parameters, determined from the EPR spectra collected (fig. 3). These are the partition parameter $F$ :

$$
F=\frac{H}{H+P}
$$

where $H$ and $P$ are explained in fig. 3(a), and the rotational correlation time $\tau$ :

$$
\tau=5.95 \cdot \Delta B \cdot\left(\sqrt{\frac{I_{0}}{I_{+1}}}+\sqrt{\frac{I_{0}}{I_{-1}}}-2\right) \cdot 10^{-10}[\mathrm{~s}]
$$

where the parameters $I_{0}, I_{-1}, I_{+1}$ and $\Delta B$ are explained in fig. 3(b). Relative measurement errors yielded $2 \%$ for the $F$ parameter and $3 \%$ for the $\tau$ one.

Preliminary tests as well as previous investigations proved the phthalocyanine dopants occupy solely the central part of the membrane, hence the choice of the 16DOXYL spin label [21]. In contrast, the TEMPO probe was useful in monitoring the bilayer periphery, fig. 4 .

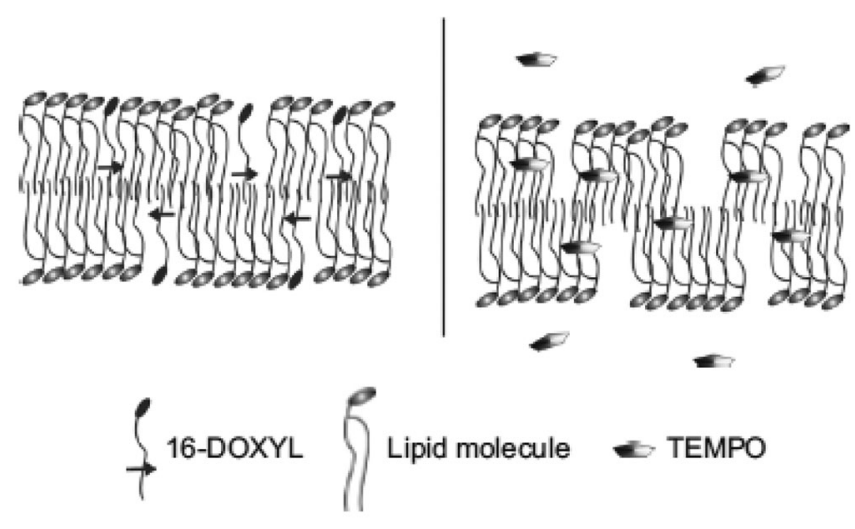

Fig. 4. Distribution of the 16-DOXYL and TEMPO spin labels within the phospholipid bilayer; the arrow indicates the position of the spin-active oxazolidinyloxy group of the 16-DOXYL probe.

The performance of a spin probe in a phospholipid membrane is strongly related to its viscosity (fluidity), and is reflected in the EPR spectrum recorded. Based on this fact, one may use specific spectral features to investigate the condition of liposome vesicles both at the waterlipid interface and the internal part of the bilayer. The TEMPO spin label is amphiphilic and hence used to explore the membrane's interface zone. From its EPR spectrum (fig. 3(a)) the partition parameter $F$ was derived to determine the distribution of the probe between the water phase (related to $P$ ) and the lipid one (related to $H$ ), which depends on the fluidity of the phospholipid set-up at the peripheral part of the membrane [24]. Increase in $F$ corresponds to enhanced fluidity. On the other hand, 16DOXYL spin label is hydrophobic and thus penetrates the bilayer's center. Since the rotation velocity of the probe within the membrane depends on its fluidity, the $\tau$ parameter defined as the rotational correlation time was derived, based on the EPR spectrum (fig. 3(b)) [25]. Hence, decrease in $\tau$ means increase in fluidity. 

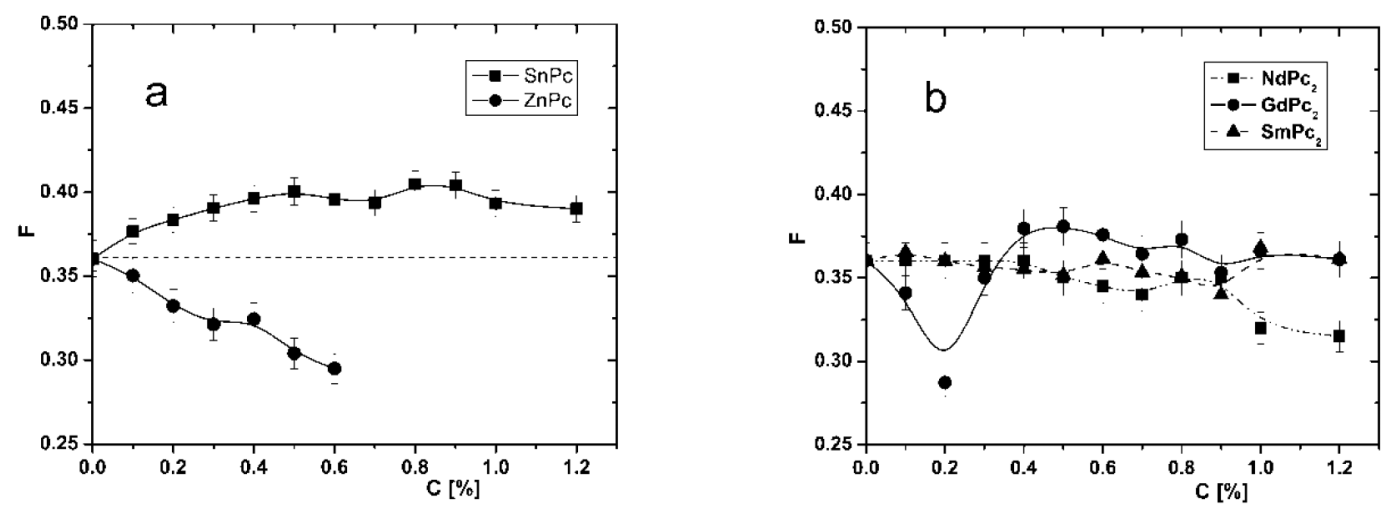

Fig. 5. Effect of the dopant concentration $C$ (\% mol, relative to EYL) on the fluidity within the peripheral layer of the EYL liposome membrane, displayed by changes in the $F$ parameter (TEMPO spin label); (a) $\mathrm{ZnPc}_{\mathrm{and}} \mathrm{SnPc}_{2}(\mathrm{~b}) \mathrm{NdPc}_{2}, \mathrm{SmPc}_{2}$ and $\mathrm{GdPc}_{2}$.

\subsection{Evaluation of static electricity}

The phthalocyanine's capability to accumulate and carry static electricity was determined by measurements of the voltage induced in contact with a platinum electrode installed inside a Faraday cage upon an insulated support. For this reason, a high-resistance electrometer Keithley6517 was applied. The sample of a dry phthalocyanine powder was placed onto the electrode which resulted in a rapid change of the registered voltage. Measurements were performed for 80 seconds each, and a gradual discharging of the electrode was observed during this period.

\section{Results and discussion}

The Pc macrocycle size fits well into a $1.1 \times 1.1 \mathrm{~nm}^{2}$ frame and the "thickness" of the sandwich molecular system in $\mathrm{LnPc}_{2}$ is about $0.43 \mathrm{~nm}$, as determined for the structures shown in fig. 1. Thus, the investigated phthalocyanines may readily diffuse into a typical liposome membrane, usually being $6-7 \mathrm{~nm}$ thick.

The effect of the phthalocyanine dopant on the $F$ parameter, as determined by the TEMPO probe, has been presented in fig. 5. These results indicate a distinct relation between the phthalocyanine molecular system and the changes in fluidity of the lipid bilayer in the vicinity of the water-membrane interface. The applied phthalocyanines were found to show a diverse activity toward the phospholipid set-up as reflected by the variation in $F$ vs. the dopant concentration. Only SnPc markedly liquidized the interface layer, as expressed by an $11 \%$ increment in $F$ relative to the reference value $\left(F_{0}\right.$, undoped liposomes), fig. 5(a). However, the other phthalocyanines apparently stiffened this layer, which follows from the decreasing value of the $F$ parameter. The most pronounced effect was displayed by $\mathrm{ZnPc}$ showing an almost $17 \%$ decrement in $F$ compared to $F_{0}$, for the dopant concentration of $0.6 \%$ in regard to EYL. Any further increase in $\mathrm{ZnPc}$ concentration resulted in a complete extinction of the $H$ line (i.e. the "lipid component", fig. 3(a)) in the EPR spectrum. The sandwich phthalocyanines ever so slightly affected the $F$ parameter, particularly up to the $0.8 \%$ dope. A somewhat larger impact was demonstrated for $\mathrm{NdPc}_{2}$ at higher concentration $(13 \%$ decrement in $F$ at $1.2 \%)$. Interestingly, for $\mathrm{GdPc}_{2}$ an unpredicted sharp decrease in $F(18.6 \%)$ appeared at concentration $0.2 \%$ which would indicate a significant stiffening of the membrane superficial layer (fig. 5(b)). However, larger amounts of this dopant produced a slight fluidizing effect relative to $F_{0}$. Such behavior may be associated with introducing uncompensated static electricity into the lipid bilayer by $\mathrm{GdPc}_{2}$ species. Similar changes in $F$ were reported previously for liposomes intercalated by chlorides $[17,18]$ and also demonstrated in computer simulations of a superficial layer of ion-doped membranes [26,27].

The changes in fluidity within the central part of the phospholipid system have been expressed by the relation of $\tau$ vs. the phthalocyanine concentration, fig. 6 and fig. 7 . These results clearly prove that all additives have been installed mainly in the middle of the membrane, consequently increasing its internal fluidity, as follows from the considerable decrease in $\tau$ by raising the concentration of the dopant. Compared to the initial rotational parameter $\left(\tau_{0}\right)$ produced by the 16-DOXYL spin label for dopant-free liposomes, the following sequence of additives according to the relative (\%) decrement in $\tau$ has been found, ZnPc $(86 \%)>\operatorname{GdPc}_{2}(60 \%)>\mathrm{NdPc}_{2}=\operatorname{SmPc}(53 \%)>\mathrm{SnPc}$ $(48 \%)$; values given in parentheses refer to the dopant concentration of $1.2 \%$. Essentially, results obtained for both independent spin probes are in good agreement with each other and may be related to the molecular set-up of the dopant. The very pronounced impact of the $\mathrm{ZnPc}$ additive reflected by quite rapid changes in $F$ and $\tau$ apparently follows from its flat structure, which allows for the relative easy penetration of the liposome interior and distribution of the species therein. This might have also explained the "stiffening effect" within the interface layer (fig. 5(a)) as the result of the rather quick diffusion of $\mathrm{ZnPc}$ molecules into the inside of the membrane with probably no particles left in the peripheral layers of the phospholipid bilayer. Finally, a more compact arrangement of the phospholipid 

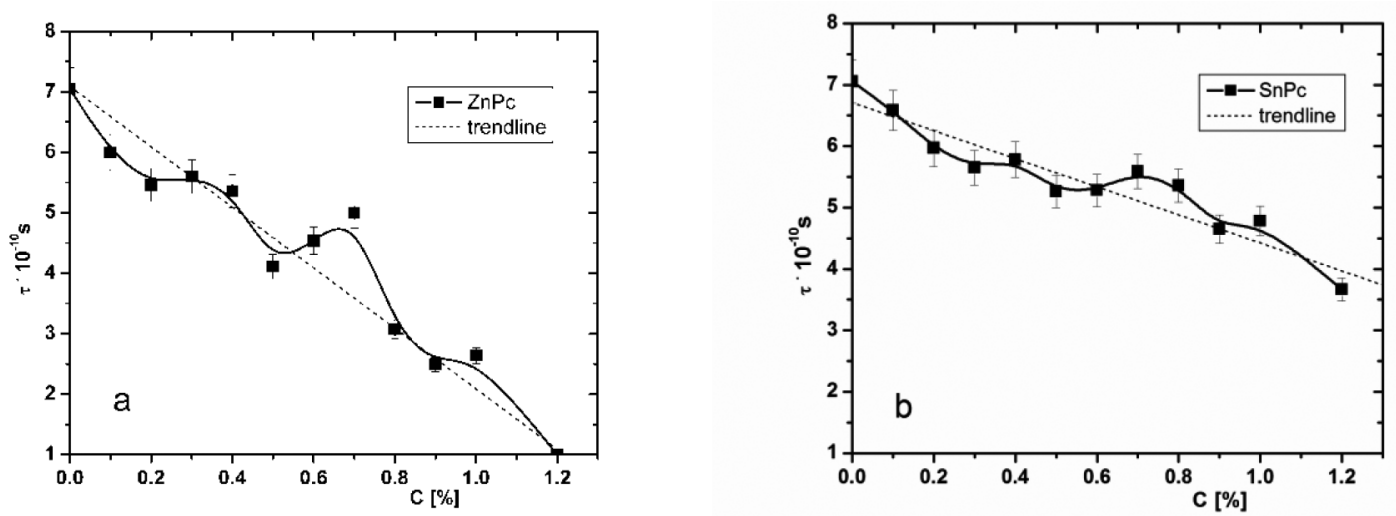

Fig. 6. Effect of the dopant concentration $C$ (\% mol, relative to EYL) on the fluidity within the central part of the EYL liposome membrane, as featured by the $\tau$ parameter (16-DOXYL spin label); (a) ZnPc and (b) SnPc.
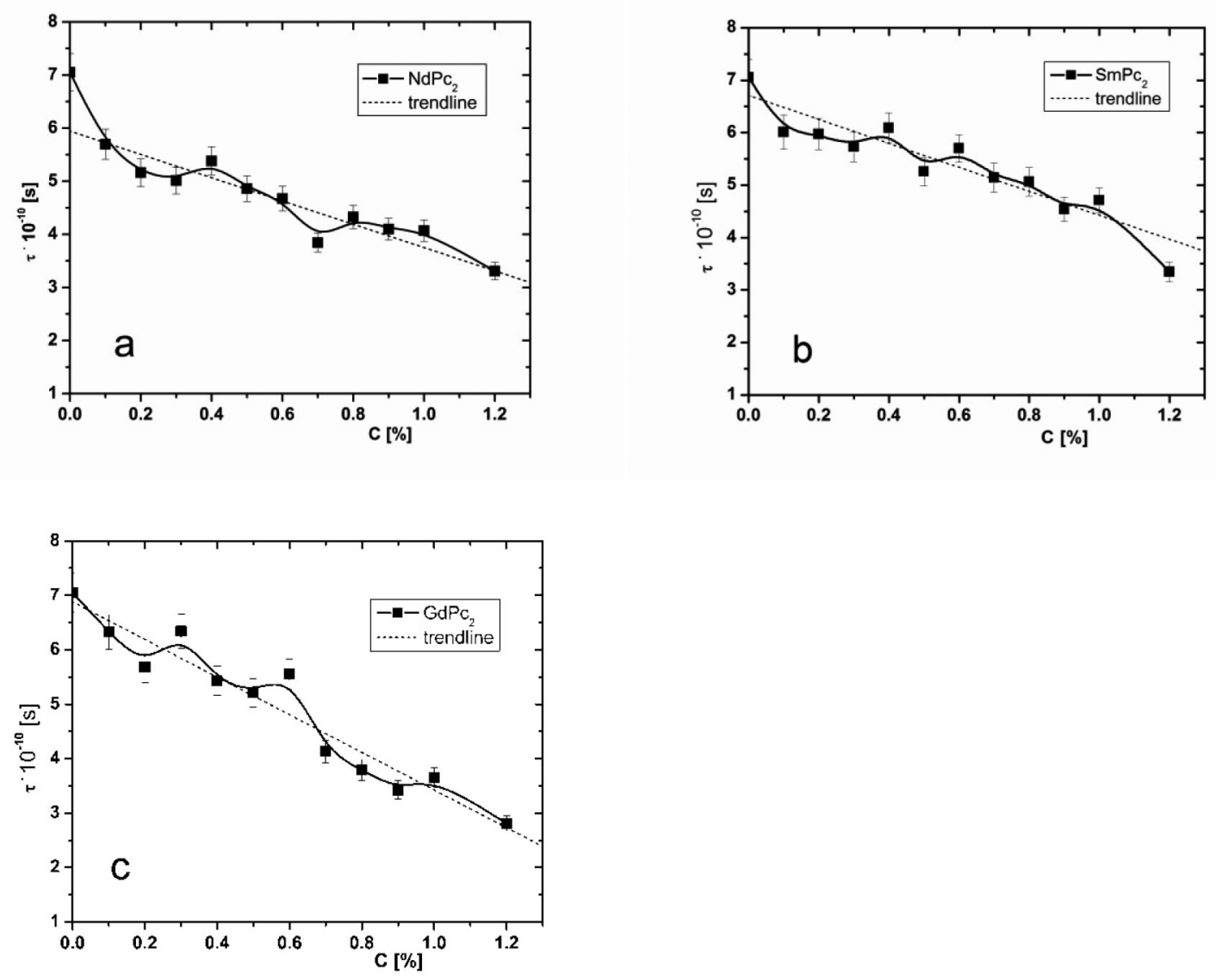

Fig. 7. Effect of the dopant concentration $C$ (\% mol, relative to EYL) on the fluidity within the central part of the EYL liposome membrane, as featured by the $\tau$ parameter (16-DOXYL spin label); (a) $\mathrm{NdPc}_{2}$, (b) $\mathrm{SmPc}_{2}$ and (c) $\mathrm{GdPc}_{2}$.

heads could be formed and, on the other hand, a more loose structure of the lipid's fatty acid tails (fig. 6(a)) featured by the decreasing values of the $F$ and $\tau$ parameters, respectively. In contrast, $\mathrm{SnPc}$ represents a funnellike molecular structure, since the $\mathrm{Sn}^{2+}$ ion does not fit into the phthalocyanine "window" determined by the four internal nitrogen atoms, hence sticking out of the macrocycle plane (fig. 1(b)). Apparently, such species penetrate the membrane more slowly than those of $\mathrm{ZnPc}$ do, and regardless of the progressive diffusion towards the center (fig. 6(b)), there is still a number of $\mathrm{SnPc}$ molecules left in the interface layer, effectively increasing its fluidity (fig. 5(a)).

The sandwich $\mathrm{LnPc}_{2}$ additives due to their more bulky molecular structure probably show lower mobility than does $\mathrm{ZnPc}$, nevertheless they also effectively penetrate the internal part of the bilayer, particularly the $\mathrm{GdPc}_{2}$ compound, fig. $7(\mathrm{c})$. Interestingly, $\mathrm{LnP}_{2}$ molecules seem to 

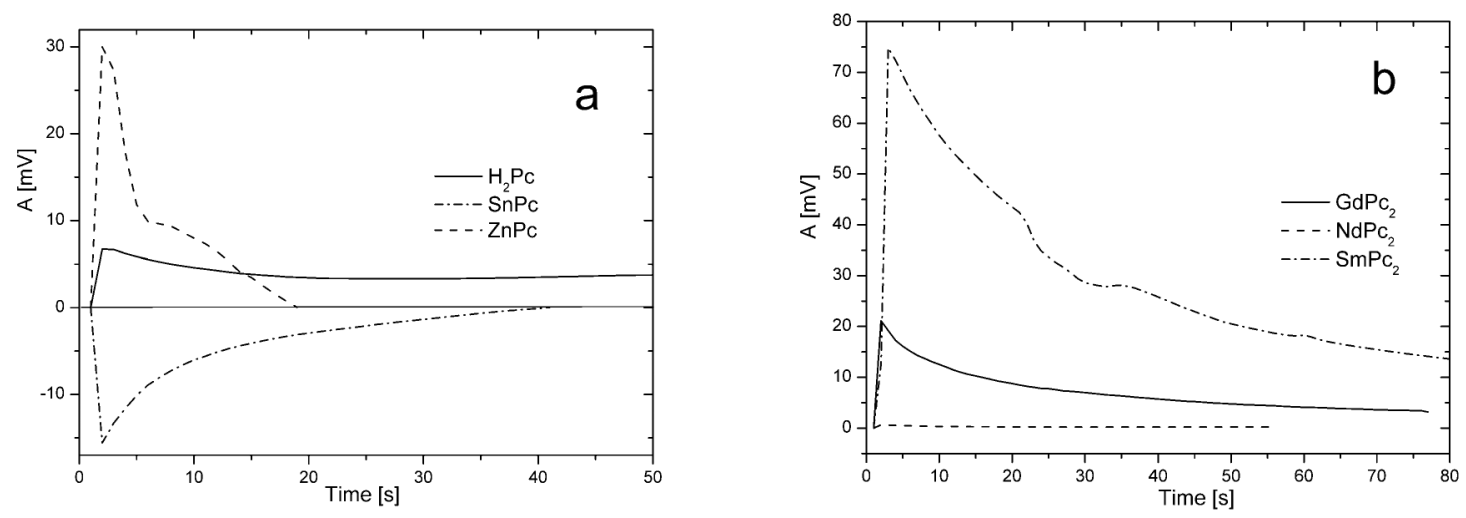

Fig. 8. Experimental evaluation of static electricity carried by the studied phthalocyanine additives $\left(\mathrm{H}_{2} \mathrm{Pc}\right.$ is the metal-free phthalocyanine); results refer to a normalized quantity of the dopant, $1 \times 10^{-5}$ mol, (a) $\mathrm{H}_{2} \mathrm{Pc}, \mathrm{SnPc}_{\mathrm{ZnPc}} \mathrm{Znd}$ (b) GdPc $\mathrm{Zn}_{2}$, $\mathrm{NdPc}_{2}, \mathrm{SmPc}_{2}$.

be installed within the liposome system definitely easier than the $\mathrm{SnPc}$ ones, as follows from the respective decrease in $\tau$. However, apart from the diverse stereochemistry, which evidently controls the molecular transport throughout the phospholipid system, phthalocyanines may carry some static electrical charge that could have produced additional effects contributing to the membrane's fluidity. In fact, only the $\mathrm{SmPc}_{2}$ compound showed a marginal static charge, whereas the other phthalocyanines demonstrated a specified static electricity carried by their solids. This was estimated based on the measurements of the voltage induced by the compounds in contact with a platinum electrode (sect. 2.3), fig. 8 .

As follows from fig. 8, only $\mathrm{SnPc}$ carried a negative charge, whereas the other compounds all featured positive static electricity, including the metal-free $\mathrm{H}_{2} \mathrm{Pc}$ (used as reference in electrical measurements but not explored in liposomes). Interestingly, $\mathrm{GdPc}_{2}$ induced a considerable higher voltage than the remaining phthalocyanines. These results are in good agreement with EPR investigations and may reasonably explain the particular behavior of $\mathrm{SnPc}$ within the interface liposome layer, in contrast to ZnPc, as well as the peculiar "stiffening effect" featured by $\mathrm{GdPc}_{2}$ (fig. 5(b)). As already mentioned, such phenomena may arise from the effect of static electricity upon the structure of the lipid bilayer [17, 18,27-31] Incidentally, organic stannous compounds have been recognized for their destructive impact upon biological membranes [32] as well as both unicellular and multicellular organisms [33].

\section{Conclusions}

The spin probe EPR technique has proved very convenient in exploring model biological membranes based on phosphatidylcholine derivatives, and appeared particularly useful in estimating the physical condition of liposome vesicles. The stability of the phospholipid set-up is crucial to its applicability in intelligent drug delivery systems, i.a. designed for transporting photoactive phthalocyanines for the reason of PDT treatment. Never- theless, different phthalocyanines once allowed to penetrate the lipid bilayer may diversely affect its fluidity and thus its stability. Although this is usually related to structural features, however one must not exclude the impact of static electricity often carried by metallophthalocyanines. Hence, synergistic effects may also account for the condition of intercalated liposomes, as evidently proved for $\mathrm{SnPc}$ showing both a stressed molecular structure (fig. 1(b)) and negative static charge (fig. 8(a)). Following the results obtained in this study, none of the dopants but $\mathrm{SnPc}$ have influenced the membrane in a particularly negative way, since these compounds have not effectively increased the fluidity of the interface layer. The fluidizing effect observed within the central part of the membrane must not be harmful at all, however when combined with the increased fluidity of the interface part, as in the case of $\mathrm{SnPc}$, then it may suggest a possible severe damage to the liposome vesicle integrity. Therefore, one must be aware when considering stannous phthalocyanine compounds for medical applications.

\section{Author contribution statement}

Dariusz Man performed EPR and electrical measurements and elaborated the results, created the graphics and compiled the article. Rudolf Słota synthetized the phthalocyanines, elaborated and discussed the EPR results and wrote the text of the article. Anna Kawecka performed EPR measurements. Grzegorz Engel performed electrical measurements and discussed the results. Gabriela Dyrda synthetized the phthalocyanines.

Open Access This is an open access article distributed under the terms of the Creative Commons Attribution License (http://creativecommons.org/licenses/by/4.0), which permits unrestricted use, distribution, and reproduction in any medium, provided the original work is properly cited. 


\section{References}

1. C.C. Leznoff, A.B.P. Lever (Editors), Phthalocyanines: Properties and Applications, Vols. 1-4 (Wiley-VCH, New York, 1989-1996).

2. R. Weiss, J. Fischer, Lanthanide phthalocyanine complexes, in The Porphyrin Handbook, edited by K. Kadish, K.M. Smith, R. Guilard, 1st edition (Academic Press Inc, New York, 2006).

3. J. Jiang (Editor), Functional Phthalocyanine Molecular Materials, in Structure and Bonding, edited by D.M.P. Mingos, Vol. 135 (Springer-Verlag, Heidelberg, 2010).

4. J.R. Darwent, P. Douglas, A. Harriman, G. Porter, M.C. Richoux, Coord. Chem. Rev. 44, 83 (1982).

5. R. Bonnett, Chem. Soc. Rev. 24, 19 (1995).

6. C.A. de Oliveira, A.E.H. Machado, F.B.T. Pessine, Chem. Phys. Lipids 133, 69 (2005).

7. A. Molnar, R. Dědic, A. Svoboda, J. Hala, J. Mol. Struct. 834-836, 488 (2007).

8. A. Salvati, S. Ristori, D. Pietrangeli, J. Oberdisse, L. Calamai, G. Martini, G. Ricciardi, Biophys. Chem. 131, 43 (2007).

9. M.N. Sibata, A.C. Tedesco, J.M. Marchetti, Eur. J. Pharm. Sci. 23, 131 (2004).

10. F. Postigo, F. Mora, M.A. De Madariaga, S. Nonell, M.L. Sagrista, Int. J. Pharm. 278, 239 (2004).

11. E. Reddi, J. Photochem. Photobiol. B 37, 189 (1997).

12. G. Falcioni, R. Gabiannelli, A. Santini, G. Zolese, D. Griffits, E. Bertoli, Appl. Organomet. Chem. 10, 451 (1996).

13. B.H. Gray, M. Porvaznik, C. Flemming, L.H. Lee, Cell. Biol. Toxicol. 3, 23 (1987).
14. H. Kleszczyńska, J. Sarapuk, S. Przestalski, Folia Histochem. Cytobiol. 37, 1 (1999).

15. D. Man, M. Podolak, G. Engel, Cell. Mol. Biol. Lett. 11, 56 (2006).

16. D. Man, I. Pisarek, M. Braczkowski, P. Pytel, R. Olchawa, J. Liposome Res. 24, 112 (2014).

17. M. Podolak, D. Man, Cell. Mol. Biol. Lett. 7, 961 (2002).

18. M. Podolak, G. Engel, D. Man, Z. Naturforsch. 61c, 453 (2006).

19. D. Man, M. Podolak, Z. Naturforsch. 62c, 427 (2007).

20. D. Man, J. Liposome Res. 18, 225 (2008).

21. D. Man, R. Słota, M.A. Broda, G. Mele, J. Li, J. Biol. Inorg. Chem. 16, 173 (2011).

22. G. Mele, E. García-López, L. Palmisano, G. Dyrda, R. Słota, J. Phys. Chem. C 111, 6581 (2007).

23. W.S. Singleton, M.S. Gray, M.L. Brown, J.L. White, J. Am. Oil Chem. Soc. 42, 53 (1965).

24. E.J. Shimshick, H.M. McConnell, Biochemistry 12, 2351 (1973).

25. M.A. Hemminga, Chem. Phys. Lipids 32, 323 (1983).

26. D. Man, R. Olchawa, K. Kubica, J. Liposome Res. 20, 211 (2009).

27. D. Man, R. Olchawa, J. Liposome Res. 23, 327 (2013).

28. M. Podolak, D. Man, S. Waga, S. Przestalski, Z. Naturforsch. 51c, 853 (1996).

29. K. Kubica, Comput. Chem. 26, 351 (2002).

30. K. Kubica, Cell. Mol. Biol. Lett. 7, 971 (2002).

31. D. Man, R. Olchawa, Eur. Biophys. J. 46, 325 (2017).

32. E. Boniewska-Bernacka, D. Man, R. Slota, M.A. Broda, J. Biochem. Mol. Toxicol. 25, 231 (2011).

33. S. Mitrus S, D. Man, J. Biochem. Mol. Toxicol. 26, 162 (2012). 